\title{
APPLICATION OF THE SPECTRAL ANALYSIS FOR MODELING
} THE ROTATIONS OF THE MOON

\author{
V.V. Pashkevich, G.I. Eroshkin \\ Central (Pulkovo) Astronomical Observatory of RAS \\ Pulkovskoe shosse, 65/1, 196140, St.Petersburg, Russia \\ e-mails: pashvladvit@yandex.ru , eroshkin@gao.spb.ru
}

\begin{abstract}
The main purposes of this research are the development of the optimal spectral analysis schemes for the investigation of the rotational motion of the Moon and then the comparison between the result of the optimal spectral analysis of the rotational motions of the Earth and the Moon. Dynamics of the rotational motion of the Moon is studied numerically by using Rodrigues-Hamilton parameters over 418.9 year time interval. The results of the numerical solution of the problem are compared with the composite semi-analytical theory of the Moon rotation (SMR) represented by Cassini relations and the semi-analytical solutions of the lunar physical libration problem (Eckhardt, 1981), (Moons, 1982), (Moons, 1984), (Pešek, 1982). The initial conditions of the numerical integration are taken from SMR. The investigation of the discrepancies is carried out by the optimal spectral analysis methods for the Newtonian case. All the periodic terms representing the behavior of the residuals are interpreted as corrections to SMR semi-analytical theory. As a result, the Moon Rotation Series (MRS2010) is constructed, which is dynamically adequate to the DE200/LE200 ephemeris over 418.9 year time interval. A numerical solution for the Moon rotation is obtained anew with the new initial conditions calculated by means of MRS2010. The discrepancies between the new numerical solution and MRS2010 do not surpass 20 mas over 418.9 year time interval. The result of the comparison demonstrates that MRS2010 series represent more accurately the Moon rotation than SMR series.
\end{abstract}

Keywords: the rotation of the Moon, ephemeris, least-squares method, spectral analysis.

\section{INTRODUCTION}

In the previous research (Pashkevich and Eroshkin, 2005) the optimal spectral analysis scheme was developed (with respect to the computation time and the accuracy) for the investigation of the rigid Earth rotation over 2000 year time interval. It is ALGORITHM-A (presented in Figure 3A) for elaboration of the very long time rigid Earth rotation series SMART97 (Bretagnon et al., 1998), which includes about 5000 periodic and Poisson terms.

In this research the optimal spectral analysis scheme is elaborated for the investigation of the Moon rotation problem and then the comparison is performed between this optimal spectral analysis scheme and ALGORITHM-A. 


\section{MATHEMATICAL MODEL OF THE PROBLEM}

The numerical solution of the problem is obtained by solving the Lagrange differential equations of the second kind for the Moon rotation with respect to the fixed ecliptic and equinox of epoch J2000. The mathematical model of the problem is described in detail in the paper (Eroshkin, Pashkevich, 1997).

Lagrange differential equations of the second kind

$$
\frac{d}{d t} \frac{\partial L}{\partial \dot{\lambda}_{i}}-\frac{\partial L}{\partial \lambda_{i}}=0, \quad i=0,1,2,3,
$$

are solved, where $L=T+U, T$ is the kinetic energy of the rotational motion of the Moon, $U$ is the force function of the gravitational interaction of the Moon with the disturbing bodies (the Earth, the Sun and the major planets). The force function $U$ is expanded in the spherical harmonics and only the terms with the coefficients $C_{j 0}$ for $j=2,3,4, C_{22}, C_{3 k}, S_{3 k}$ for $\mathrm{k}=1,2,3, \mathrm{C}_{4 \mathrm{k}}, \mathrm{S}_{4 \mathrm{k}}$ for $\mathrm{k}=1, \ldots, 4$ are used. The orbital motions of the disturbing bodies are defined by the DE200/LE200 ephemeris.

The Rodrigues - Hamilton parameters

$\lambda_{0}=\cos \frac{\theta}{2} \cos \frac{\psi+\varphi}{2}, \lambda_{1}=\sin \frac{\theta}{2} \cos \frac{\psi-\varphi}{2}, \lambda_{2}=\sin \frac{\theta}{2} \sin \frac{\psi-\varphi}{2}, \lambda_{3}=\cos \frac{\theta}{2} \sin \frac{\psi+\varphi}{2}$

are the functions of the Euler angles $\psi, \theta$ and $\varphi$. The Rodrigues - Hamilton parameters are bounded variables. It is very important for the numerical solution of the problem.

The semi-analytical theory of the Moon rotation (SMR) consists of Cassini relations and the semi-analytical solution of the lunar physical libration problem.

1. Cassini's relations:

a) The Moon rotates with a constant angular velocity around its polar axis. The rotation period is equal to the mean sidereal period of its orbital motion around the Earth:

$$
\varphi_{d}+\psi_{d}=180^{\circ}+L
$$

b) The inclination of the lunar equator to the ecliptic is a constant angle (close to $1^{\mathrm{O}} 32^{\prime}$ ):

$$
\theta_{d}=I \text {. }
$$

c) The ascending node of the lunar orbit on the ecliptic coincides with the descending node of the lunar equator on the ecliptic:

$$
\psi_{d}=\Omega \text {. }
$$

In the above equalities $\psi_{d}$ is the longitude of the descending node of date of the lunar equator, $\theta_{d}$ is the inclination of the lunar equator to the ecliptic of date, $\varphi_{d}$ is the proper rotation angle between the descending node of date and the principal axis of the minimum moment of inertia, $I$ is the mean inclination of the lunar equator to the ecliptic of date, $L$ is the mean longitude of the Moon and $\Omega$ is the mean longitude of the ascending node of its orbit. date.

Figure 1 depicts the variables, which are used to define Euler angles for the ecliptic of 


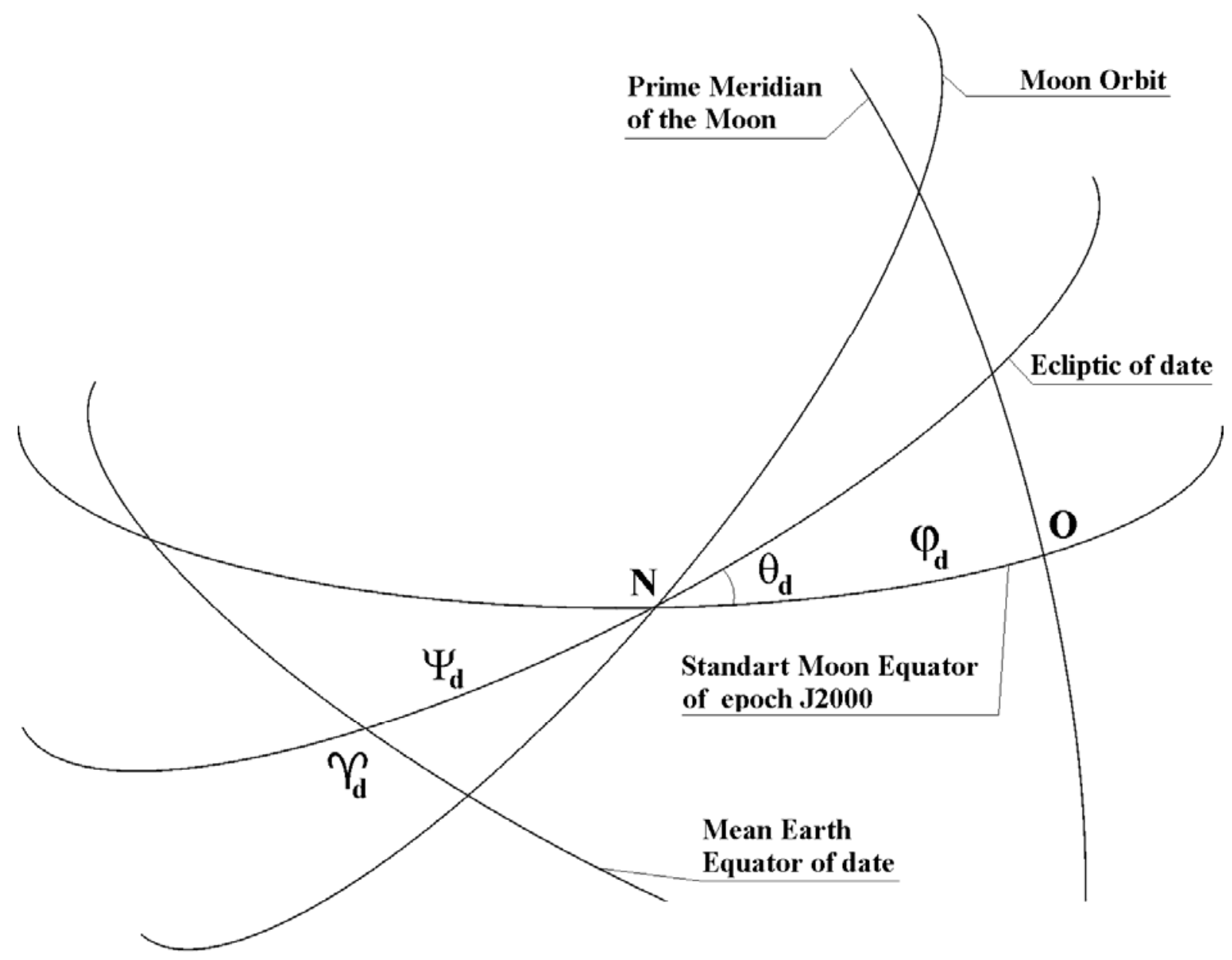

Fig. 1. Definition of Euler angles for the ecliptic of date

Since Cassini's relations are not exact, one must consider the perturbed Euler angles:
a) $\varphi_{d}+\psi_{d}=180^{\circ}+L+\tau$,
b) $\theta_{d}=I+\rho$,
c) $\psi_{d}=\Omega+\sigma$,

where $\tau, \rho$ and $\sigma$ are the perturbing terms of the physical librations in the longitude, in the inclination and in the node longitude, respectively.

2. The semi-analytical solution of the lunar physical libration problem includes four solutions:

a) MP500 (Moons, 1982) is the 3rd degree solution for the force function $\left(C_{j 0}\right.$ for $j=2,3, C_{22}$, $\mathrm{C}_{3 \mathrm{k}}, \mathrm{S}_{3 \mathrm{k}}$ for $\left.\mathrm{k}=1,2,3\right)$.

b) Additional solution (Eckhardt, 1981) for the 4th degree perturbations of the force function $\left(\mathrm{C}_{40}, \mathrm{C}_{4 \mathrm{k}}, \mathrm{S}_{4 \mathrm{k}}\right.$ for $\left.\mathrm{k}=1, \ldots, 4\right)$. 
c) Effect of the planetary perturbations (Moons, 1984).

d) Effect of the Earth's flattening on the rotation of the Moon (Pešek, 1982). (1982):

The expressions for the main terms of the solutions of Eckhardt (1981) and Moons

$$
\left.\begin{array}{l}
\tau_{m}=\sum_{k}\left[\tau_{S k} \sin \left(v_{k 0}+v_{k 1} t\right)+\tau_{C k} \cos \left(v_{k 0}+v_{k 1} t\right)\right] \\
p_{1 m}=\sum_{k}\left[p_{1 S k} \sin \left(v_{k 0}+v_{k 1} t\right)+p_{1 C k} \cos \left(v_{k 0}+v_{k 1} t\right)\right] \\
p_{2 m}=\sum_{k}\left[p_{2 S k} \sin \left(v_{k 0}+v_{k 1} t\right)+p_{2 C k} \cos \left(v_{k 0}+v_{k 1} t\right)\right]
\end{array}\right\},
$$

where $v_{k 0}, v_{k l}$ are the phases and the frequencies of SMR theory, respectively, $t$ is the time in the Julian days.

The expressions for the planetary perturbations and the terms due to the flattening of the Earth (Moons, 1984), (Pešek, 1982):

$$
\left.\begin{array}{l}
\tau_{p}=\sum_{j} q_{j} \sin \left(v_{j 0}+v_{j 1} t\right) \\
p_{1 p}=\sum_{j}\left[q_{2 j} \cos \left(v_{j 0}+v_{j 1} t\right) \sin F+q_{1 j} \sin \left(v_{j 0}+v_{j 1} t\right) \cos F\right] \\
p_{2 p}=\sum_{j}\left[q_{2 j} \cos \left(v_{j 0}+v_{j 1} t\right) \cos F-q_{1 j} \sin \left(v_{j 0}+v_{j 1} t\right) \sin F\right]
\end{array}\right\}
$$

where $F=L-\Omega$.

Finally, the expressions for the terms of the Moon rotation solution (SMR) are

$$
\left.\begin{array}{l}
\tau=\tau_{m}+\tau_{p} \\
p_{1}=p_{1 m}+p_{1 p} \\
p_{2}=p_{2 m}+p_{2 p}
\end{array}\right\}
$$

The expressions for the Euler angles with respect to the ecliptic of date:

$$
\begin{aligned}
& \tan \varphi_{d}=\frac{p_{1}}{p_{2}}, \\
& \sin \theta_{d}=\sqrt{p_{1}^{2}+p_{2}^{2}}, \\
& \psi_{d}=180^{\circ}+L+\tau-\varphi_{d} .
\end{aligned}
$$




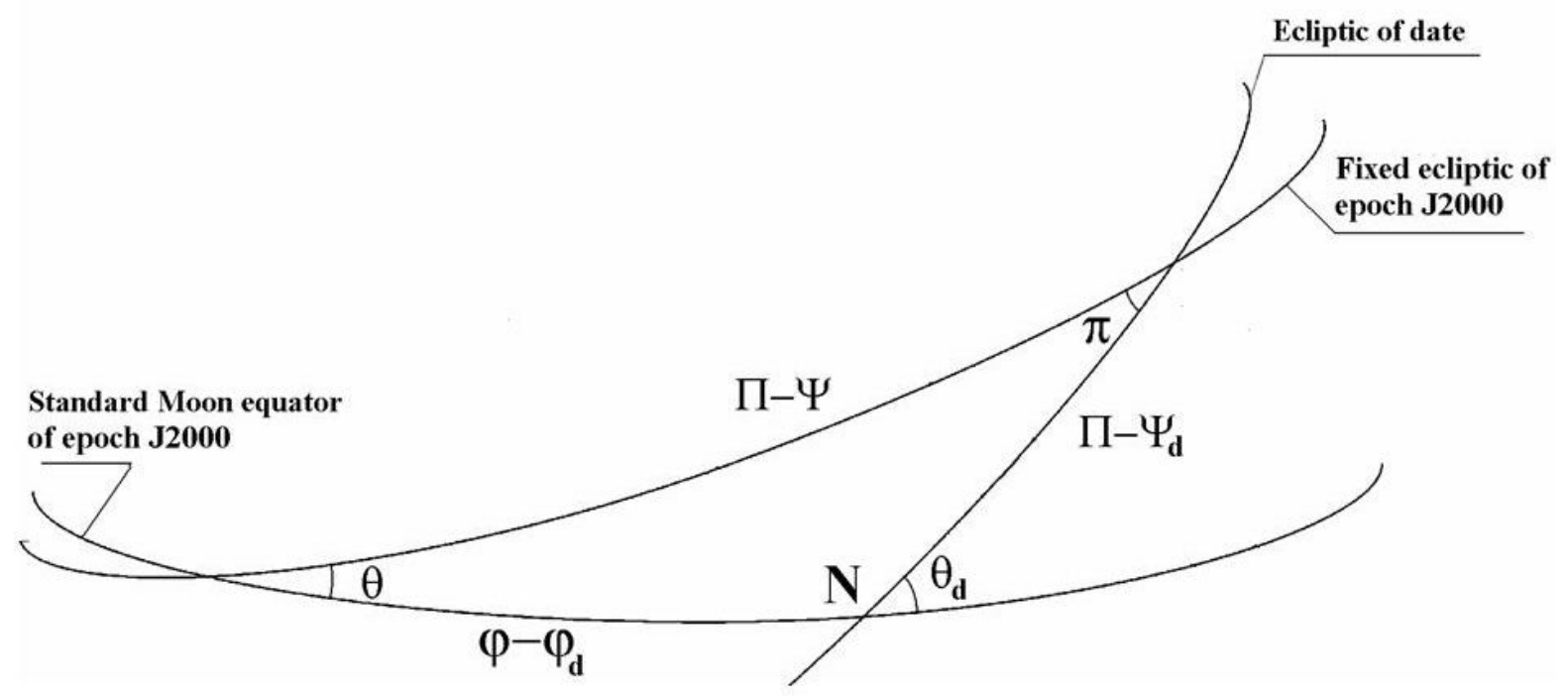

Fig. 2. Basic triangle for the reduction of Euler angles

The following formulae are applied for the reduction of Euler angles from the ecliptic of date to the fixed ecliptic of epoch J2000 for the Moon rotation (see Figure 2):

$$
\begin{aligned}
& \cos \theta=\cos \theta_{d} \cos \pi+\sin \theta_{d} \sin \pi \cos \left(\Pi-\psi_{d}\right), \\
& \sin \left(\varphi-\varphi_{d}\right)=\frac{\sin \pi \sin \left(\Pi-\psi_{d}\right)}{\sin \theta}, \\
& \sin \left(\psi-\psi_{d}\right)=-\frac{\cos \theta+\cos \theta_{d} \sin \left(\varphi-\varphi_{d}\right)}{1+\cos \pi}, \\
& \dot{\theta}=\dot{\theta}_{d} \cos \left(\varphi-\varphi_{d}\right)-\dot{\pi} \cos (\Pi-\psi)+\left(\dot{\Pi}-\dot{\psi}_{d}\right) \sin \pi \sin (\Pi-\psi), \\
& \dot{\varphi}-\dot{\varphi}_{d}=\frac{\left(\dot{\Pi}-\dot{\psi}_{d}\right) \sin \pi \cos (\Pi-\psi)+\dot{\pi} \sin (\Pi-\psi)-\dot{\theta}_{d} \sin \left(\varphi-\varphi_{d}\right) \cos \theta}{\sin \theta} \\
& \dot{\Pi}-\dot{\psi}=\frac{\left(\dot{\Pi}-\dot{\psi}_{d}\right) \sin \theta_{d} \cos \left(\varphi-\varphi_{d}\right)+\dot{\pi} \sin (\Pi-\psi) \cos \theta-\dot{\theta}_{d} \sin \left(\varphi-\varphi_{d}\right)}{\sin \theta} .
\end{aligned}
$$

In the above expressions $\theta$ is the inclination of the lunar equator to the fixed ecliptic $\mathrm{J} 2000, \psi$ is the longitude of the descending node of epoch J2000 of the lunar equator, $\varphi$ is the proper rotation angle between the descending node of epoch J2000 and the principal axis of the minimum moment of inertia.

The expressions for the classical perturbing terms (Newhall and Williams, 1997) of the physical librations:

$$
\left.\begin{array}{l}
\Delta \tau=\sum_{j} \sum_{k=0}^{3}\left[\tau_{S j k} \sin \left(v_{j 0}+v_{j 1} t\right)+\tau_{C j k} \cos \left(v_{j 0}+v_{j 1} t\right)\right] t^{k} \\
\Delta \rho=\sum_{j} \sum_{k=0}^{3}\left[\rho_{S j k} \sin \left(v_{j 0}+v_{j 1} t\right)+\rho_{C j k} \cos \left(v_{j 0}+v_{j 1} t\right)\right] t^{k} \\
\Delta I \sigma=I \sum_{j} \sum_{k=0}^{3}\left[\sigma_{S j k} \sin \left(v_{j 0}+v_{j 1} t\right)+\sigma_{C j k} \cos \left(v_{j 0}+v_{j 1} t\right)\right] t^{k}
\end{array}\right\} .
$$


As a result of compilation, a semi-analytical theory of the Moon rotation is constructed with respect to the ecliptic and the equinox of date. Then the semi-analytical theory is reduced to the fixed ecliptic J2000.0.

The orbital motions of the disturbing bodies are defined by the DE200/LE200 ephemeris. The high-precision numerical integration method (Belikov, 1990), with a number of modifications (Eroshkin et al., 1993), was applied.

\section{ALGORITHMS AND RESULTS}

The result of the comparison between the numerical solution and semi-analytical solution SMR is studied by means of two variants of the spectral analysis schemes (presented in Figure $3 \mathrm{~A}$ and Figure 3B).

Spectral analysis (SA) method (A L G O R I T H M-A)

for removal of the periodical terms from the discrepancies

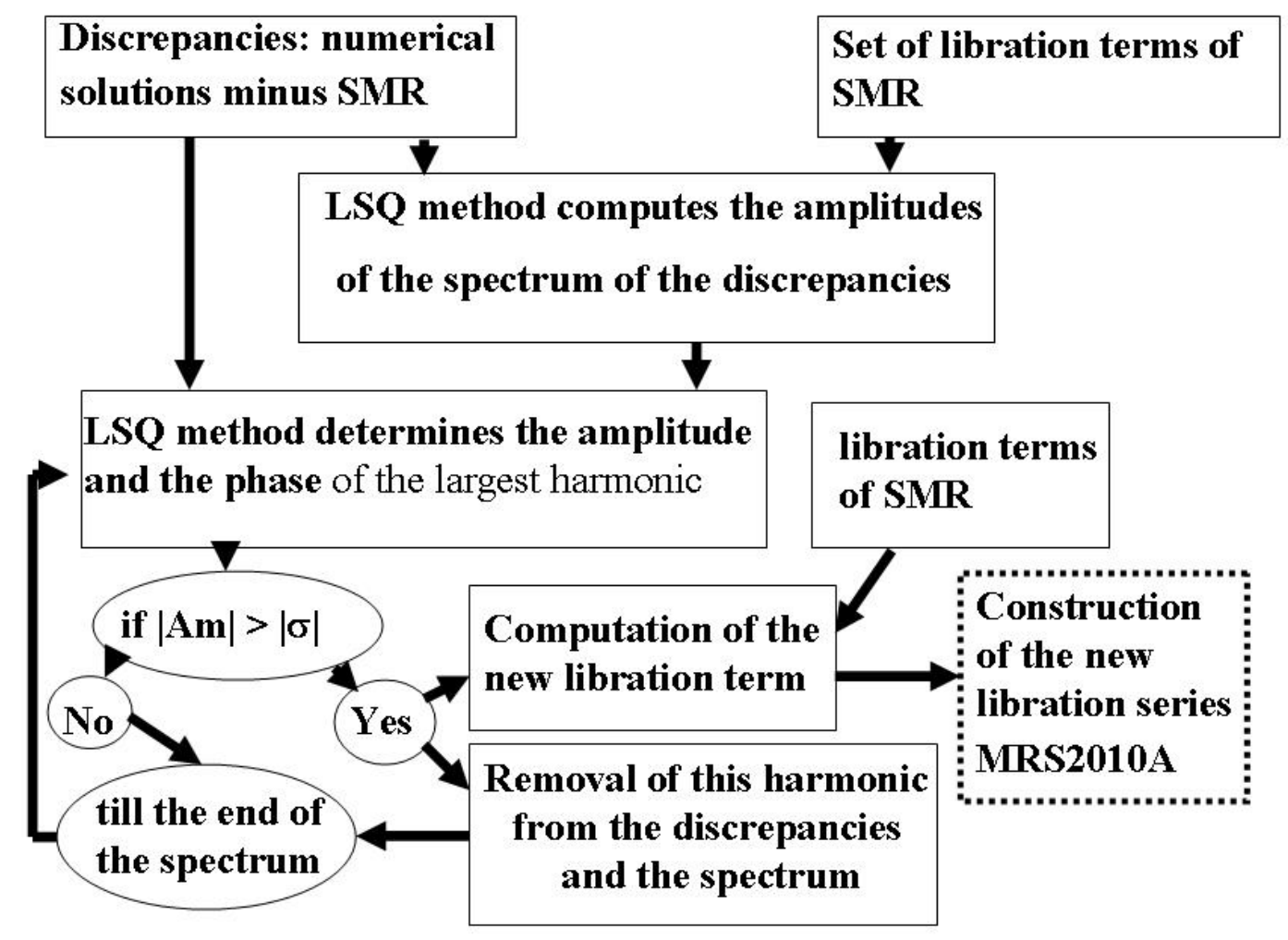

Fig. 3A. Algorithm-A 
Spectral analysis (SA) method (A L G O R I T H M-B)

for removal of the periodical terms from the discrepancies
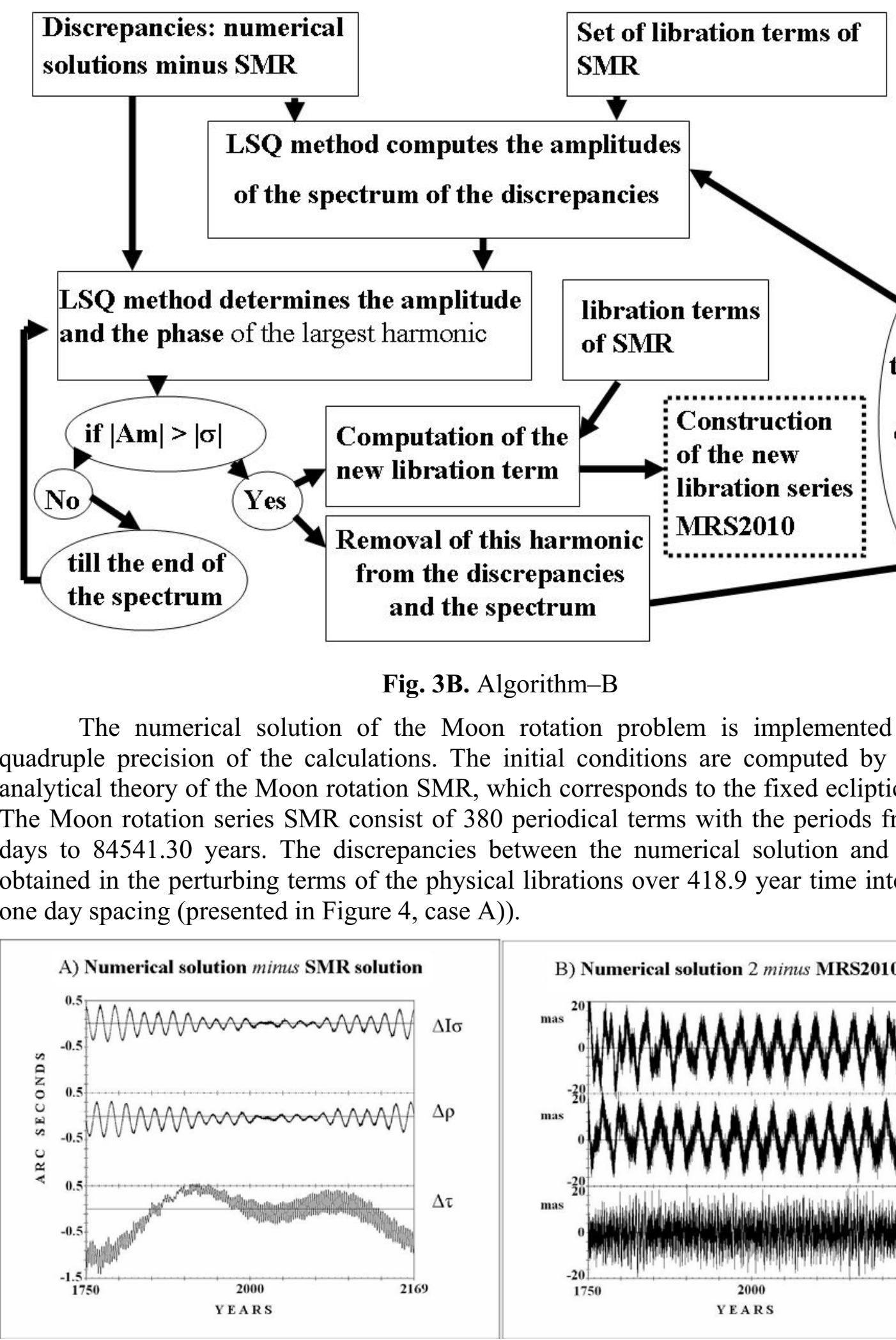
The investigation of the discrepancies is carried out by the least squares method (LSQ) and by the spectral analysis method (SA). The set of the frequencies of SMR theory is used without a change. Only the coefficients of the periodic terms are improved.

SA method-A: The spectrum of the discrepancies between the numerical solution and SMR is constructed only once (presented in Figure 5, ALGORITHM-A). Every coefficient of the new periodic term equals the sum of the calculated periodic term coefficient and the coefficient of the corresponding periodic term of SMR. The found new harmonic is removed from the discrepancies and from the spectrum. Starting from the maximum term of the spectrum the procedure is accomplished successively up to its least term. The new periodic and Poisson terms representing the new series MRS2010A are determined.

SA method-B: The spectrum of the discrepancies is constructed anew after the removal of every largest residual harmonic from the discrepancies. Each constructed spectrum is used for the determination of the new coefficient of the periodic term as in SA method-A. This procedure is performed for every harmonic of the set. The new periodic and Poisson terms representing the new series MRS2010 are determined.

The compilation of the power spectrum of the discrepancies between the numerical and semi-analytical SMR solutions for ALGORITHM-B is constructed, beginning from the largest of the residual harmonics, step by step (presented in Figure 5, ALGORITHM-B).

The power spectra represented in Figure 5 are restricted by the terms with the periods from 5.648 days to 418.9 years. Therefore the set of the frequencies of SMR theory, which is used in SA methods, is restricted to only 351 periodical terms.

Figure 5 demonstrates that the composed power spectrum of the discrepancies between the numerical and semi-analytical SMR solutions for ALGORITHM-B is more preferable than the power spectrum for ALGORITHM-A, because the close harmonics distinguish more clearly from each other (presented in Figure 5, ALGORITHM-B).

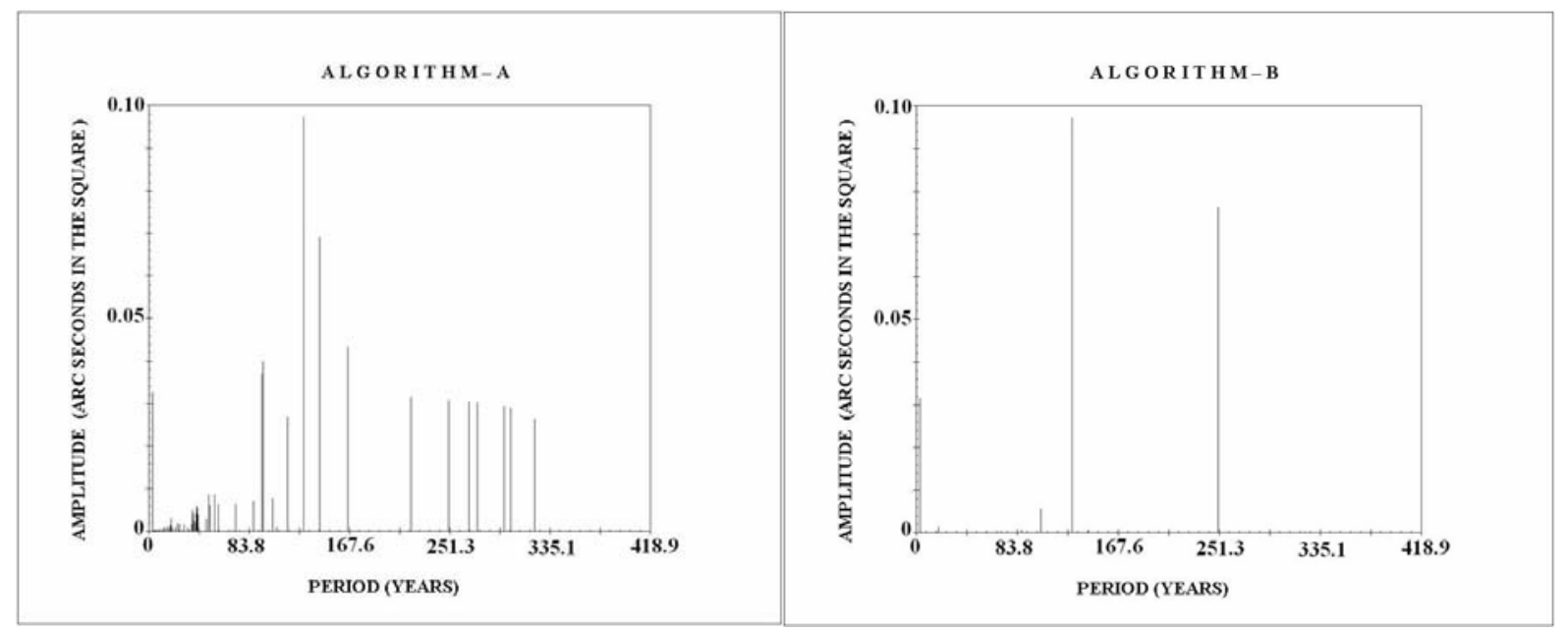

Fig. 5. Spectrum of the discrepancies between the numerical and SMR solutions for $\Delta \tau$

Figure 6 demonstrates that the residuals, after the formal removal of 351 periodical terms from the discrepancies between the numerical and semi-analytical SMR solutions, for ALGORITHM-B are less than the ones for ALGORITHM-A.

This investigation demonstrates that ALGORITHM-B is more accurate than ALGORITHM-A. Thus MRS2010 is better than MRS2010A.

Then the numerical solution for the Moon rotation is constructed anew with the new initial conditions calculated by means of MRS2010, which includes 1433 periodical and 
Poisson terms. The appearance of Poisson terms in the harmonics is the main difference between MRS2010 series and SMR series.

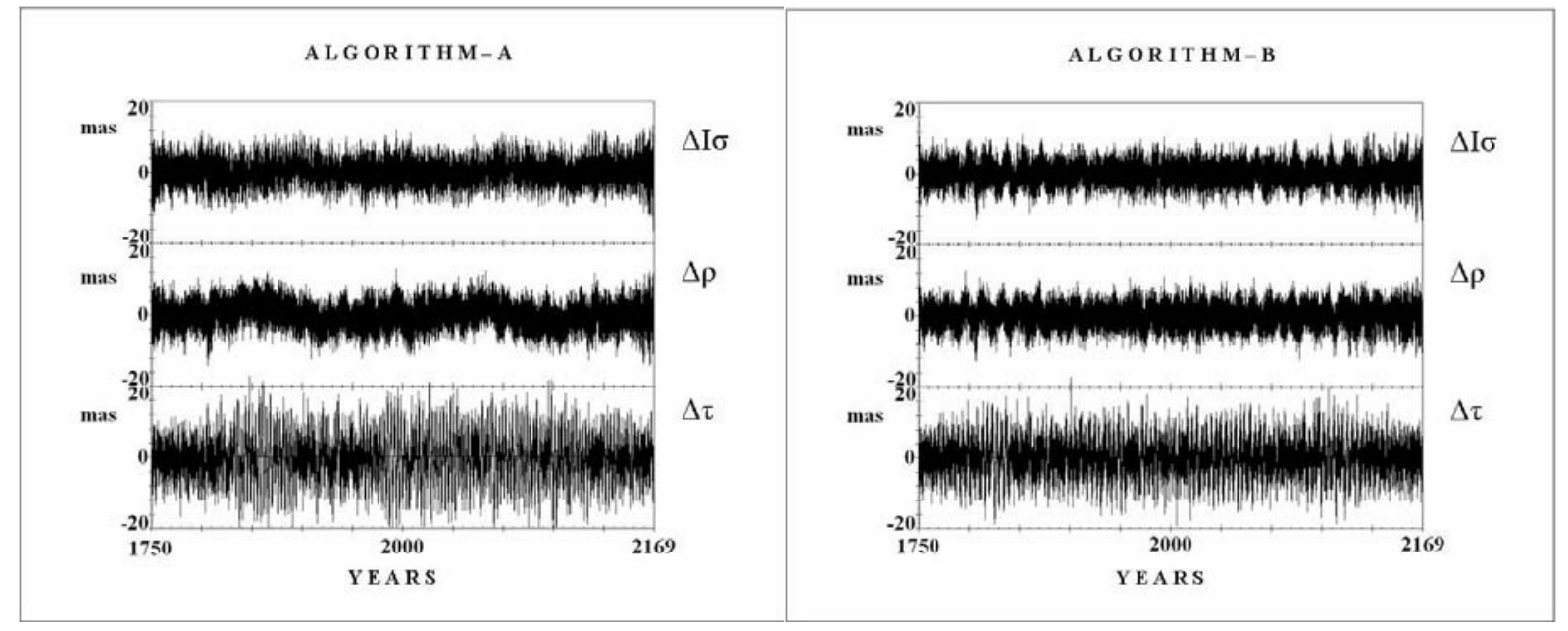

Fig. 6. Residuals after the formal removal of 351 periodical terms from the discrepancies between the numerical and semi-analytical (SMR) solutions

The results of the comparison of the new numerical integration of the lunar rotation with the new MRS2010 are presented in Figure 4, case B). The residuals do not reveal secular trends. The behavior of the residuals can be described by the superposition of the harmonics representing both the forced physical libration and the fictive free physical libration. The most essential harmonics of the residuals are the periodic harmonics of the fictive free physical libration with the period of 2.9 years in the longitude $(\Delta \tau)$, with the periods of 27.2 days and of 24 years in the inclination $(\Delta \rho)$ and in the node longitude $(\Delta \mathrm{I} \sigma)$. The appearance of the harmonics of the fictive free physical libration in the numerical solution is explained by the errors of the initial conditions, which are due to the limited accuracy of the used semianalytical solutions.

Figure 4 demonstrates that the discrepancies of the comparison in the libration angles decrease 25 times for both $\Delta \mathrm{I} \sigma$ and $\Delta \rho$, and 75 times for $\Delta \tau$, after the construction of the new more accurate MRS2010 semi-analytical solution.

The discrepancies of the comparison between the numerical solutions and MRS2010 do not surpass 20 mas over 418.9 year time interval (presented in Figure 4B). It means an essentially better consistency of MRS2010 series with the DE200/LE200 ephemeris.

\section{CONCLUSIONS}

It was found that ALGORITHM-B, in which the spectrum is constructed anew after the removal of every largest residual harmonic from the discrepancies, is more accurate than ALGORITHM-A, in which the spectrum is constructed only one time. The execution time ratio between ALGORITHM-B and ALGORITHM-A depend on the number of the time series terms. If $T_{A}-$ execution time for ALGORITHM-A, where the spectrum is constructed only one time for $N$ terms of time series, and $T_{B}-$ execution time for ALGORITHM-B, where the spectrum is constructed $N$ ones for $N$ terms of time series. Then $T_{B} \approx N T_{A}$.

ALGORITHM-B is very time-consuming. Hence it is not suitable for the investigation of the long time series (which includes around 5000 and more terms), as follows from our previous investigation of the Earth rotation (Pashkevich and Eroshkin, 2005). However ALGORITHM-B is very suitable for the investigation of the short time series (the Moon rotation). 
The new more accurate series MRS2010, dynamically adequate to the DE200/LE200 ephemeris over 418.9 year time interval, is constructed. MRS2010 includes 1433 periodical and Poisson terms.

The discrepancies between the numerical solution and MRS2010 do not surpass 20 mas over 418.9 year time interval.

\section{ACKNOWLEDGEMENTS}

The investigation was carried out at the Central (Pulkovo) Astronomical Observatory of the Russian Academy of Science and the Space Research Centre of the Polish Academy of Science, under a financial support of the Cooperation between the Polish and Russian Academies of Sciences, Theme No 38 and the grant of Polish Academy of Science, No $52603732 / 3972$.

\section{REFERENCES}

Belikov M.V. (1990): Methods of numerical integration with uniform and mean square approximation for solving problems of ephemeris astronomy and satellite geodesy, Manus. Geod., 15, No4, pp.182-200.

Bretagnon P., Francou G., Rocher P., Simon J.L. (1998): SMART97: A new solution for the rotation of the rigid Earth, A\&A 329, pp. 329-338.

Eckhardt D.H. (1981): Theory of Libration of the Moon, The Moon and the planets, 25, pp.349.

Eroshkin G.I., Taibatorov K.A., Trubitsina A.A. (1993): Constructing the specialized numerical ephemerides of the Moon and the Sun for solving the problems of the Earth's artificial satellite dynamics, ITA RAS, Preprint No 31, (in Russian).

Eroshkin G.I., Pashkevich V.V. (1997): Numerical Simulation of the Rotational Motion of the Earth and Moon, Dynamics and Astrometry of Natural and Artificial Celestial Bodies, IAU Colloquium 165 (I.M.Wytrzyszczak, J.H.Lieske, R.A.Feldman, eds), Kluwer, Dordrecht, pp.275-280.

Moons M. (1982): Physical Libration of the Moon, Celest. Mech., 26, pp.131-142.

Moons M. (1984): Planetary Perturbations on the Libration of the Moon, Celest. Mech., 34, pp.263-273.

Pashkevich V.V., Eroshkin G.I. (2005): Choice of the optimal spectral analysis scheme for the investigation of the Earth rotation problem, in Proc. of Journees 2005: Earth dynamics and reference systems: five years after the adoption of the IAU 2000 Resolutions (Space Research Centre of Polish Academy of Sciences, Warsaw, Poland, 19-21 September 2005), pp. 105-109.

Pešek I. (1982): An Effect of the Earth's Flattening on the Rotation of the Moon, Bull. Astron. Inst. Czechosl., 33, pp.176-179.

Newhall X X and Williams J.G. (1997): Estimation of the lunar physical librations, Celestial Mechanics and Dynamical Astronomy 66, pp. 21-30.

Received: 2010-12-16,

Reviewed: 2010-01-27,

Accepted: 2010-03-01. 